\title{
Sparse Control Using Sum-of-norms Regularized Model Predictive Control
}

\author{
Sina Khoshfetrat Pakazad, Henrik Ohlsson and Lennart Ljung
}

\section{Linköping University Post Print}

\section{* Tweet}

N.B.: When citing this work, cite the original article.

(C)2013 IEEE. Personal use of this material is permitted. However, permission to reprint/republish this material for advertising or promotional purposes or for creating new collective works for resale or redistribution to servers or lists, or to reuse any copyrighted component of this work in other works must be obtained from the IEEE.

Sina Khoshfetrat Pakazad, Henrik Ohlsson and Lennart Ljung, Sparse Control Using Sum-ofnorms Regularized Model Predictive Control, 52nd IEEE Conference on Decision and Control, 2013.

Postprint available at: Linköping University Electronic Press

http://urn.kb.se/resolve?urn=urn:nbn:se:liu:diva-103914 


\title{
Sparse Control Using Sum-of-norms Regularized Model Predictive Control
}

\author{
Sina Khoshfetrat Pakazad, Henrik Ohlsson and Lennart Ljung
}

\begin{abstract}
Some control applications require the use of piecewise constant or impulse-type control signals, with as few changes as possible. So as to achieve this type of control, we consider the use of regularized model predictive control (MPC), which allows us to impose this structure through the use of regularization. It is then possible to regulate the trade-off between control performance and control signal characteristics by tuning the so-called regularization parameter. However, since the mentioned trade-off is only indirectly affected by this parameter, its tuning is often unintuitive and time-consuming. In this paper, we propose an equivalent reformulation of the regularized MPC, which enables us to configure the desired trade-off in a more intuitive and computationally efficient manner. This reformulation is inspired by the so-called $\varepsilon$ constraint formulation of multi-objective optimization problems and enables us to quantify the trade-off, by explicitly assigning bounds over the control performance.
\end{abstract}

\section{INTRODUCTION}

Sparsity has been an important topic in estimation in recent years. The origin for the current interests can be traced to the Lasso algorithm, [1], which trades off the number of estimated parameters in linear regressions to the model fit. This is achieved by adding a regularization term to the criterion of fit which penalizes the number of nonzero elements. That idea has been used for a large variety of problems in system identification, signal processing and signal representation, see e.g., [2]-[4]. An advanced theory of sparsity and compressed sensing has been developed in [5], [6].

At the same time, in control theory, there has long been a wish to not only curb the size of the input, like in various optimization problems, but also restrict the number of actual control actions, to spare control equipment. So called Lebesgue sampling has been suggested to schedule control actions, [7]. The paper [8], for example, contained examples on how to achieve good tracking or trajectory generation with as few control interventions as possible.

The authors gratefully acknowledge support by the Swedish Department of Education within the ELLIIT project, the Swedish Research Council in the Linnaeus center CADICS, the European Research Council under the advanced grant LEARN, contract 267381, by a postdoctoral grant from the Sweden-America Foundation, donated by ASEA's Fellowship Fund, and by a postdoctoral grant from the Swedish Research Council.

Khoshfetrat Pakazad, Ohlsson and Ljung are with the Division of Automatic Control, Department of Electrical Engineering, Linköping University, Sweden. Ohlsson is also with the Department of Electrical Engineering and Computer Sciences, University of California, Berkeley, CA, USA. sina.kh.pa@isy.liu.se, ohlsson@isy.liu.se, ljungeisy.liu.se.
In this contribution Model Predictive Control (MPC) will be studied from a similar perspective. MPC has become a leading control paradigm in industrial practice in the past decades. It will be shown that similar regularization terms added to the standard MPC criterion will achieve sparseness in control action. The trade-off between control accuracy and the number of control actions is, as in the estimation case, decided by the size of the regularization parameter. This is a major tuning problem and a substantial part of this contribution deals with rational choices for how this can be solved using multi-objective optimization.

This paper is organized as follows. In Section II, we formulate the MPC problem and briefly review basic concepts in MPC. Section III, provides a description of the regularized model predictive control with sum-of-norms regularization and discusses the importance of proper tuning of the socalled regularization parameter. In Section IV, we formulate an alternative formulation of the regularized MPC, and illustrate the effectiveness of the proposed regularized MPC formulation through some numerical examples in Section V. We conclude the paper with some final remarks in Section VI.

\section{Linear Quadratic Model Predictive Control}

In this paper, we assume that the state dynamics of the system to be controlled is described by

$$
x(k+1)=A x(k)+B u(k)
$$

where $A \in \mathbb{R}^{n \times n}$ and $B \in \mathbb{R}^{n \times m}$. Given $x_{0}$, we intend to find a control sequence that is the minimizing argument of the following optimization problem

$$
\begin{aligned}
\operatorname{minimize} & \sum_{k=1}^{\infty}\left[\begin{array}{c}
x(k) \\
u(k-1)
\end{array}\right]^{T} \mathbf{Q}\left[\begin{array}{c}
x(k) \\
u(k-1)
\end{array}\right]+q^{T} x(k)+r^{T} u(k-1) \\
\text { subj. to } & x(k+1)=A x(k)+B u(k) \quad k=0,1, \cdots \\
& C_{x} x(k)+C_{u} u(k) \leq d, \quad k=0,1, \cdots \\
& x(0)=x_{0},
\end{aligned}
$$

where $u(0), u(1) \cdots, x(0), x(1) \cdots$ are the optimization variables, $C_{x} \in \mathbb{R}^{p \times n}, C_{u} \in \mathbb{R}^{p \times m}$ and $\mathbf{Q}=\left[\begin{array}{cc}Q & S \\ S^{T} & R\end{array}\right] \succeq$ 0 , with $Q \succeq 0$ and $R \succ 0$. The cost function (defined by data matrices $\mathbf{Q}, q, r)$ is usually chosen based on control performance specifications and the linear equality and inequality constraints describe the feasible operating region 
of the system. This control strategy is referred to as the infinite horizon optimal control, [9], and requires the solution to an infinite-dimensional optimization problem. In order to avoid solving this infinite dimensional optimization problem, often a suboptimal heuristic for solving the problem in (2), is considered. This heuristic approach is referred to as the model predictive control (MPC). In this heuristic method the horizon of the control problem is truncated to a finite value, $H$, and instead a receding horizon strategy is undertaken [9], [10]. This means that, the control action at each time step $t$ and given $x(t)$, is obtained by first solving the following optimization problem

$$
\begin{aligned}
& \operatorname{minimize} \sum_{k=0}^{H-1}\left[\begin{array}{c}
x(k) \\
u(k-1)
\end{array}\right]^{T} \mathbf{Q}\left[\begin{array}{c}
x(k) \\
u(k-1)
\end{array}\right]+q^{T} x(k)+r^{T} u(k-1) \\
& \quad+x(H)^{T} Q_{H} x(H)+q_{H}^{T} x(H) \\
& \text { subj. to } x(k+1)=A x(k)+B u(k), \quad k=0,1, \cdots H-1 \\
& C_{x} x(k)+C_{u} u(k) \leq d, \quad k=1, \cdots, H-1 \\
& C_{H} x(H) \leq d_{H}, \quad C_{0} u(0) \leq d_{0} \\
& x(0)=x(t),
\end{aligned}
$$

where $u(0), \cdots, u(H-1), x(0), \cdots, x(H)$ are the optimization variables, $C_{H} \in \mathbb{R}^{p_{H} \times n}, C_{0} \in \mathbb{R}^{q_{0} \times m}$ and $Q_{H} \succeq 0$. Solving this optimization problem results in the optimal solution, $u^{*}(0), \cdots, u^{*}(H-1)$ and $x^{*}(1), \cdots, x^{*}(H)$. The MPC controller then, as the next control input to the system, uses $u(t)=u^{*}(0)$ and repeats the same procedure at time step $t+1$ for the starting point $x(t+1)$. Note that the formulation in (3), also covers other classical presentations of MPC, [10]. In order to guarantee stability and recursive feasibility in the receding horizon strategy, $C_{H}, d_{H}, Q_{H}$ and $q_{H}$ have to be chosen with care, [9]-[13]. In the following we assume that these matrices are chosen accordingly so that we would not have any concerns about the stability and feasibility issues of the problem in (3).

The problem in (3) defines a quadratic program (QP), which is convex and can be solved efficiently, using for instance interior point methods, [14]. Particularly, it was shown in [15] that solving the problem in (3) using interior point methods would require $\mathcal{O}\left(H(m+n)^{3}\right)$ floating point operations (flops). If the data matrices that define this QP are chosen properly, then MPC generates control input sequences that will guarantee stability, will be feasible and will produce satisfactory control performance. However, we are interested in control inputs that have special structures, particularly control signals that are either piecewise constant with small number of changes or have impulse-type format uniformly in all channels, and it is not possible to tune these data matrices to produce control actions with these structures. One of the possible remedies for this issue is through the use of nonsmooth regularization. This is discussed in Section III.

\section{Regularized Model Predictive Control}

The use of regularizing terms for inducing sparsity or other special structures on solutions of optimization problems has been previously used in other areas such as signal processing, system identification, etc, [1], [5], [16]-[19], and has also recently found interest in control, [8], [20], [21]. In this paper we also employ a similar strategy for inducing special structure or sparsity on the generated control signals. To this end, we modify the cost function of the problem in (3) as

$$
\begin{aligned}
& \sum_{k=0}^{H-1}\left[\begin{array}{c}
x(k) \\
u(k-1)
\end{array}\right]^{T} \mathbf{Q}\left[\begin{array}{c}
x(k) \\
u(k-1)
\end{array}\right]+q^{T} x(k) \\
& +r^{T} u(k-1)+x(H)^{T} Q_{H} x(H)+q_{H}^{T} x(H) \\
& +\lambda\left\|\left(\left\|R_{1} U\right\|_{p}, \ldots,\left\|R_{r} U\right\|_{p}\right)\right\|_{0}
\end{aligned}
$$

where $U=(u(0), \ldots, u(H-1))$. The additional term in the cost function penalizes the existence of non-zero elements in vector $\left(\left\|R_{1} U\right\|_{p}, \ldots,\left\|R_{r} U\right\|_{p}\right)$ with penalty parameter $\lambda$. We refer to this parameter as the regularization parameter, and it sets the trade-off between the control performance and desired control signal characteristics. We refer to this problem as the regularized MPC. Note that by choosing $R, \lambda$ and $p$ properly, regularized MPC will provide us with a control signal sequence that satisfies our predefined specifications. Introducing the $\ell_{0}$-norm, however, changes the underlying optimization problem from a convex minimization problem into a non-convex one. In order to circumvent this issue, the $\ell_{0}$-norm is often approximated by its convex envelop which is the $\ell_{1}$-norm, [1], [5], [16], [18]. By using this approximation, (4) can be rewritten as

$$
\begin{array}{r}
\sum_{k=0}^{H-1}\left[\begin{array}{c}
x(k) \\
u(k-1)
\end{array}\right]^{T} \mathbf{Q}\left[\begin{array}{c}
x(k) \\
u(k-1)
\end{array}\right]+q^{T} x(k)+r^{T} u(k-1) \\
\quad+x(H)^{T} Q_{H} x(H)+q_{H}^{T} x(H)+\lambda \sum_{i=1}^{r}\left\|R_{i} U\right\|_{p},
\end{array}
$$

and as a result, the underlying optimization problem in regularized MPC can be formulated as

$$
\begin{array}{ll}
\operatorname{minimize} & \sum_{k=0}^{H-1}\left[\begin{array}{c}
x(k) \\
u(k-1)
\end{array}\right]^{T} \mathbf{Q}\left[\begin{array}{c}
x(k) \\
u(k-1)
\end{array}\right]+q^{T} x(k)+r^{T} u(k-1) \\
& +x(H)^{T} Q_{H} x(H)+q_{H}^{T} x(H)+\lambda \sum_{i=1}^{r}\left\|R_{i} U\right\|_{p} \\
\text { subj. to } & x(k+1)=A x(k)+B u(k), \quad k=0,1, \cdots H-1 \\
& C_{x} x(k)+C_{u} u(k) \leq d, \quad k=1, \cdots, H-1 \\
& C_{H} x(H) \leq d_{H}, \quad C_{0} u(0) \leq d_{0} \\
& x(0)=x(t) .
\end{array}
$$

This formulation was first proposed in [8] and later discussed under the name $\ell_{\text {asso }}$ MPC in [20]. The most common choices of regularization term are with $p=1,2$, which are the so-called $\ell_{1}$-norm and sum-of-norms regularization respectively. It is known that using the $\ell_{1}$-norm regularization forces many elements in the vectors $R_{i} U, i=1, \ldots, r$, to be equal to zero. We refer to this sparsity as the element-wise sparsity. Similarly, utilizing sum-of-norms regularization will 
also generate sparsity in vectors $R_{i} U, i=1, \ldots, r$. However, unlike the $\ell_{1}$-norm regularization, this regularization term is known to produce solutions with whole vectors $R_{i} U=0$. This is the so-called group sparsity. In this paper, we focus on using the sum-of-norms regularization, i.e., $p=2$. We can use this characteristic of sum-of-norms regularization to induce piecewise constant or impulse-type properties on the computed control signals. To be more precise, let $R_{i}^{T}=$ $\left(e_{i}+e_{i+1}\right) \otimes I_{m}$ for $i=1, \ldots, H-1$, with $e_{i}$ denoting the $i$ th column of the identity matrix. Using the following regularization term we can then induce piecewise constant structure on the control inputs produced using regularized MPC,

$$
\|u(0)-u(t-1)\|_{2}+\sum_{i=1}^{H-1}\|u(i-1)-u(i)\|_{2},
$$

where $u(t-1)$ is the last used input (the input used at time step $t-1)$. The use of the first term in (7) is for penalizing the difference between the produced control inputs at different time steps. Using this regularization term, also punishes the variations of the control signal within the horizon $H$. Consequently, the controller tends to use piecewise constant control signals with minimal number of changes. Similarly, let $R_{i}^{T}=e_{i} \otimes I_{m}$, which will result in the regularization term $\sum_{i=0}^{H-1}\|u(i)\|_{2}$. This choice of regularization term penalizes the use of nonzero control inputs, and will hence produce control signals that mimic the behavior of impulse control. Note that due to the use of $\ell_{2}$-norm, at each time step, the mentioned structures would appear uniformly in all $m$ control channels. This will not be the case if one chooses to use $\ell_{1}$ norm regularization.

It goes without saying that the choice of regularization parameter, $\lambda$, affects the resulting solution, and achieving a satisfactory solution requires performing rigorous tuning of this parameter, at each time step. As was mentioned before, this parameter describes the trade-off between the two competing terms in the objective value, which correspond to the control performance and the desired control structure. Despite the importance of proper tuning of this parameter, this problem is not given enough attention and is currently done by ad-hoc procedures, e.g., in signal processing applications see [17], [22], that can be quite time consuming, counter-intuitive and cumbersome. This is because by changing this parameter, we can only make qualitative predictions regarding the yielded trade-off, and in order to study quantitative properties of the resulting trade-off we would need to solve the corresponding optimization problem. Consequently, tuning $\lambda$, such that the controller would provide us with a satisfactory result, would require solving the underlying optimization problem several times (at each time step). This can become computationally costly, particularly due to the fact that solving the minimization problem in (6), with $p=2$, requires solving either a second order cone program (SOCP) or quadratically constraint QP (QCQP), [14], which can be considerably more computationally demanding than solving a QP. In Section IV, we propose an equivalent reformulation of the regularized MPC problem, that would allow us to set the desired trade-off in a more intuitive manner and hence, computationally efficient manner.

\section{A ReFormulation of The Regularized MPC}

The optimization problem in (6) can also be regarded as a multi-objective optimization problem given as

$$
\begin{array}{cl}
\operatorname{minimize} & {\left[\begin{array}{c}
l(X, U) \\
\sum_{i=1}^{r}\left\|R_{i} U\right\|_{p}
\end{array}\right]} \\
\text { subj. to } & (X, U) \in \mathcal{C}
\end{array}
$$

where $X=(x(0), \ldots, x(H))$, the set $\mathcal{C}$ represents the feasible region described in problem in (6) and $l(X, U)$ denotes the cost function for the problem in (3). The aim of this optimization problem is to minimize both terms in the objective vector, simultaneously, while satisfying the constraints. Note that in essence and originally we are interested in finding the solution to this problem, and the problem in (6) is only defined as a consequence.

One of the ways of computing the so-called Pareto optimal solutions, [14], [23], for this problem is by means of the weighted sum method which results in the formulation given in (6), [14], [23]. Such techniques intend to find all the Pareto optimal solutions by studying the solutions of the problem in (6) for all positive values of $\lambda$. By doing so one can achieve the so-called barrier frontier, [14], and can then choose the optimal solution that suits them best (usually the knee of the frontier). Although this approach is perhaps the most widely used one, it is not necessarily the best way of handling (8). This is because, the weighted sum method requires exploring the frontier barrier which can be very time consuming and counter-intuitive. There are also other methods for solving the problem in (8), (or verifying Pareto optimality of solutions for (8)), namely, Benson's method, $\varepsilon$-constraint method, hybrid methods and elastic constraint method, [23]-[26]. These methods, including the weighted sum method, belong to a wider class of multi-objective optimization approaches, called scalarization techniques, [23]. Next, we explore the possibility of utilizing the $\varepsilon$-constraint method for solving the problem in (8).

\section{A. $\varepsilon$-constraint Method}

After the weighted sum method, the $\varepsilon$-constraint method is perhaps the best known apparatus for solving multiobjective optimization problems. This method was proposed by [24], and is based on solving constrained optimization problems formed based on the original problem. Consider the following multi-objective optimization problem

$$
\underset{x \in \mathcal{X}}{\operatorname{minimize}}\left[\begin{array}{lll}
f_{1}(x) & \ldots & f_{N}(x)
\end{array}\right]^{T} .
$$


This optimization problem can be handled through solving a set of $\varepsilon$-constrained problems defined as

$$
\begin{array}{cl}
\operatorname{minimize} & f_{j}(x) \\
\text { subj. to } & f_{i}(x) \leq \varepsilon_{i} \quad i=1, \ldots, N, \quad i \neq j . \\
& x \in \mathcal{X}
\end{array}
$$

It was shown by [27], that in case $\mathcal{X}$ and all $f_{i}$ s are all convex, for any optimal solution of the problem in (10), $x^{*}$, for some $j$, there exist $\lambda_{i} \geq 0$ such that $x^{*}$ is also an optimal solution for

$$
\text { minimize } \sum_{i=1}^{N} \lambda_{i} f_{i}(x) \text {. }
$$

As a result, using this approach, one can compute the Pareto optimal solutions for the problem in (9), by changing $\varepsilon_{i} \mathrm{~s}$. Note that both the weighted sum and $\varepsilon$-constraint methods still suffer from the same problem while searching for Pareto solutions, where in order to find the desired Pareto solution one may have to perform time consuming tuning of $\lambda_{i} \mathrm{~s}$ and $\varepsilon_{i}$ s. However, for our case (i.e., the regularized MPC problem) using this method for solving the problem enables us to look for the desired solution in a much more intuitive manner. This is investigated in the following section.

\section{B. $\varepsilon$-constraint Formulation of Regularized MPC}

By following the guidelines presented in the previous section, the regularized MPC problem can be cast in the form of an $\varepsilon$-constraint problem as below

$$
\begin{array}{cl}
\underset{X, U}{\operatorname{minimize}} & \sum_{i=1}^{r}\left\|R_{i} U\right\|_{p} \\
\text { subj. to } & l(X, U) \leq \varepsilon \\
& (X, U) \in \mathcal{C}
\end{array}
$$

In this formulation the objective function only concerns the regularization of the control variables and the term concerning the control performance has been formulated as a constraint. One of the shortcomings of this formulation in comparison to the formulation in (6) is that, the problem in (12) is not necessarily feasible with respect to all choices of $\varepsilon$. In order to avoid this issue, we choose $\varepsilon=p^{*}\left(1+\epsilon_{p}\right)$ where $p^{*}$ is the optimal objective value for the problem in (3) and $\epsilon_{p}>0$ is referred to as the tolerated $\epsilon$-optimality which is a design parameter. Note that $p^{*}$ is the achieved optimal control performance, which is obtained without forcing any structure on the control input, i.e., with no regularization term. With this choice of $\varepsilon$, not only feasibility of the problem in (3) would imply feasibility of the problem in (12), but also it is possible to show that the formulations are in fact very closely related.

\section{Relation Between the Formulations of the Regularized MPC Problem}

Recall that $\mathcal{C}$ is a polyhedral constraint, and the set $\mathcal{D}=$ $\{(X, U) \in \mathcal{C} \mid l(X, U)<\varepsilon\}$ is nonempty. As a result the
Slater's constraint qualification holds and we have strong duality, [14]. Consider the KKT optimality conditions, for this problem given below

$$
\begin{aligned}
& l\left(X^{*}, U^{*}\right) \leq \varepsilon \\
& \nu^{*}(\varepsilon) \geq 0 \\
& \nu^{*}\left(l\left(X^{*}, U^{*}\right)-\varepsilon\right)=0 \\
& \left(X^{*}, U^{*}\right)=\underset{(X, U) \in \mathcal{C}}{\arg \min }\left\{\sum_{i=1}^{r}\left\|R_{i} U\right\|_{p}+\nu^{*}(l(X, U)-\varepsilon)\right\}
\end{aligned}
$$

where $\nu^{*}(\varepsilon)$ is the optimal Lagrange multiplier for the constraint in $(12 \mathrm{~b})$, and $X^{*}, U^{*}$ are the optimal primal variables. The notation $\nu^{*}(\varepsilon)$ has been used for denoting the optimal Lagrange multiplier to emphasize its dependence on the chosen $\varepsilon$. Assume that $\nu^{*}(\varepsilon)>0$. Then, by (13d), the optimal primal variables are the solutions of the following optimization problem

$$
\begin{array}{ll}
\operatorname{minimize} & \nu^{*}(\varepsilon) l(X, U)+\sum_{i=1}^{r}\left\|R_{i} U\right\|_{p} \\
\text { subj. to } & (X, U) \in \mathcal{C} .
\end{array}
$$

Note that this optimization problem is equivalent to the problem in (6), with $\lambda=1 / \nu^{*}(\varepsilon)$. Also since we have strong duality, the complementary slackness implies that $l\left(X^{*}, U^{*}\right)=\varepsilon$. This states that, the solution $\left(X^{*}, U^{*}\right)$ constitutes a $\epsilon_{p} p^{*}$-suboptimal solution for the MPC problem in (3), and shows how much comprise, with respect to control performance, had to be made to achieve the obtained control signal properties.

In case $\nu^{*}(\varepsilon)=0$, the optimality conditions in (13), imply that the optimal primal variables $\left(X^{*}, U^{*}\right)$ are obtained by solving the following optimization problem

$$
\begin{array}{ll}
\operatorname{minimize} & \sum_{i=1}^{r}\left\|R_{i} U\right\|_{p} \\
\text { subj. to } & (X, U) \in \mathcal{C} .
\end{array}
$$

Compare the problem in (6) with the one in (14). In this case, it is as though in the problem in (6), the chosen $\lambda=\infty$, and it is equivalent to neglecting the term corresponding to the control performance in the cost function. This is because even using $\left(X^{*}, U^{*}\right)$, which only minimizes the regularization term, will in the worst case be $\epsilon_{p} p^{*}$-suboptimal.

Using the formulation in (12) of the regularized MPC problem enables us to evade the tuning of the regularization parameter, and we instead would need to tune $\epsilon_{p}$. However, unlike $\lambda$, tuning $\epsilon_{p}$ is more intuitive. This is because $\epsilon_{p}$ quantitatively sets how much of the control performance we are willing to sacrifice to obtain a control input that has a certain characteristic. This is particularly beneficial, if the control designer knows what is the maximum allowed suboptimality in control performance.

Remark 1: Notice that forming the problem in (12) at each time step requires solving a $\mathrm{QP}$, that is for computing $p^{*}$, which can be solved efficiently, [13]. However, since tuning 
$\epsilon_{p}$ is more intuitive than tuning $\lambda$, at each time step, the cost of forming and solving the problem in (12) would be lower than the one in (6). This is even more evident, as in for many cases we can preset $\epsilon_{p}$ and keep it constant, since this will create a consistent trade-off between the control performance and control signal structure (as we will see in the numerical results section). Note that in order to achieve a similar behavior one still needs to diligently tune $\lambda$ at each time step.

\section{NUMERICAL EXAMPLES}

In this section, we employ the regularized MPC for controlling a drone while loitering above a designated location with a certain loitering radius, $r_{l}$, and speed, $\omega$. The aim is to stay as close as possible to the loitering circle and use piecewise constant control signal to reduce the wear of the actuators (which control the heading of the vehicle) and the sound signature of the drone. These actuators are only activated when we switch between two levels in the required control signals. As a result, using piecewise control signals with minimal number of changes, reduces the usage of these actuators and will hence reduce the possibility of failure of them while loitering for long periods of time. The discretetime state dynamics model of this system is given as below

$$
x_{s}(k+1)=\left[\begin{array}{cccc}
1 & 0 & 1 & 0 \\
0 & 1 & 0 & 1 \\
0 & 0 & 1 & 0 \\
0 & 0 & 0 & 1
\end{array}\right] x_{s}(k)+\left[\begin{array}{cc}
0.005 & 0 \\
0 & 0.005 \\
1 & 0 \\
0 & 1
\end{array}\right]\left[\begin{array}{l}
u_{1}(k) \\
u_{2}(k)
\end{array}\right],
$$

where $x_{s}(k)=\left[\begin{array}{llll}x(k) & y(k) & v_{x}(k) & v_{y}(k)\end{array}\right]^{T}$. We apply the regular MPC to this control problem, as it is stated in (3), where at each time step $t$ and given $x_{s}(t), u(t-1)$ and $x_{\text {ref }}(k)$, for $k=1, \ldots, H$, we solve the following optimization problem

$$
\begin{array}{ll}
\operatorname{minimize} & \sum_{k=1}^{H}\left(\left[\begin{array}{l}
x(k) \\
y(k)
\end{array}\right]-x_{\mathrm{ref}}(k)\right)^{T} Q\left(\left[\begin{array}{l}
x(k) \\
y(k)
\end{array}\right]-x_{\mathrm{ref}}(k)\right) \\
& +\sum_{k=1}^{H-1}(u(k)-u(k-1))^{T} R(u(k)-u(k-1)) \\
& +(u(0)-u(t-1))^{T} R(u(0)-u(t-1)) \\
\text { subj. to } \quad & x_{s}(k+1)=A x_{s}(k)+B u(k), \quad k=0,1, \cdots H-1 \\
& -50 \preceq\left[\begin{array}{l}
x(k) \\
y(k)
\end{array}\right] \preceq 50, \quad-60 \preceq\left[\begin{array}{l}
v_{x}(k) \\
v_{y}(k)
\end{array}\right] \preceq 60, \\
& k=1, \cdots, H-1 \\
& -120 \preceq u(k) \preceq 120, \quad k=0, \cdots, H-1 \\
& -20 \preceq u(k)-u(k-1) \preceq 20, \quad k=1, \cdots, H-1 \\
& x_{s}(0)=x_{s}(t),
\end{array}
$$

where $Q=10 I_{2}, R=8.45 I_{2}$ and $H=5$. Note that the tuning of these parameters have been performed meticulously, particularly to quantify the trade off between the tracking of the reference and size of changes in control input in a proper manner. The tracking reference is generated recursively at each time step, where at first the current position of the flying object is projected onto the circle centered at the designated

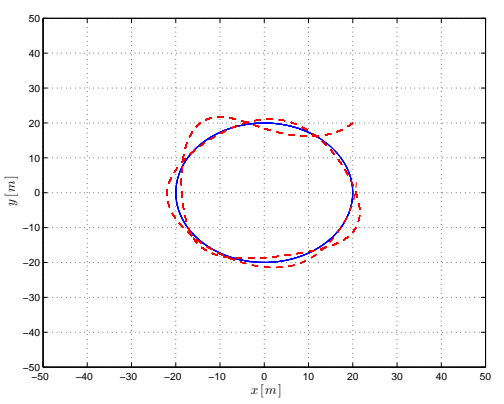

Fig. 1. The obtained tracking performance using MPC. The solid line illustrates the generated reference at each time instant, and the dashed line presents the position of the drone.

location with radius $r_{l}$, and then the reference for the next $H$ time steps is generated by simulating the movement of the object along the circle with constant angular velocity $\omega$. Figures 1 and 2, illustrate the achieved performance using this controller when $r_{l}=20$ and $\omega=1$. As can be seen from Figure 1, the controller provides a good enough tracking performance. However, the generated control signals are always changing and on average include 94 switches between consecutive values. Next, we apply the regularized MPC to this control problem. Note that, tuning $\lambda$ in (6) in order to match the same performance achieved here, requires substantial amount of time and hence, instead, we use the regularized MPC, with the following formulation

$$
\begin{array}{ll}
\text { minimize } & \|u(0)-u(t-1)\|_{2}+\sum_{i=1}^{H-1}\|u(i-1)-u(i)\|_{2} \\
\text { subj. to } & x_{s}(k+1)=A x_{s}(k)+B u(k), \quad k=0,1, \cdots H-1 \\
& -50 \preceq\left[\begin{array}{l}
x(k) \\
y(k)
\end{array}\right] \preceq 50, \quad-60 \preceq\left[\begin{array}{l}
v_{x}(k) \\
v_{y}(k)
\end{array}\right] \preceq 60, \\
& k=1, \cdots, H-1 \\
& -120 \preceq u(k) \preceq 120, \quad k=0, \cdots, H-1 \\
& -20 \preceq u(k)-u(k-1) \preceq 20, \quad k=1, \cdots, H-1 \\
& l(X) \leq \varepsilon \\
& x_{s}(0)=x_{s}(t),
\end{array}
$$

where $l(X)=\sum_{k=1}^{H}\left(\left[\begin{array}{l}x(k) \\ y(k)\end{array}\right]-x_{\mathrm{ref}}(k)\right)^{T} Q\left(\left[\begin{array}{c}x(k) \\ y(k)\end{array}\right]-x_{\mathrm{ref}}(k)\right)$. In this case we choose $\epsilon_{p}=0.3$, which means that we have decided to sacrifice $30 \%$ of the tracking performance to achieve a piecewise constant control signal. Figures 3 and 4, illustrate the performance of this controller. As can be observed form Figure 3, is even more uniform than the previous case and this has been obtained by only allowing 69 changes in the control signal, which illustrates the effectiveness of the sum-of-norms regularization. This results in $27 \%$ reduction in the usage of actuators. The reduction in the changes in control signal is particularly visible between sample intervals, 13-26, 40-60 and 78-88.

\section{CONCLUSIONS}

In this paper, we investigated the effectiveness of sum-ofnorms regularized MPC. Producing a good control performance using this control strategy, requires rigorous and timeconsuming tuning of the so-called regularization parameter 

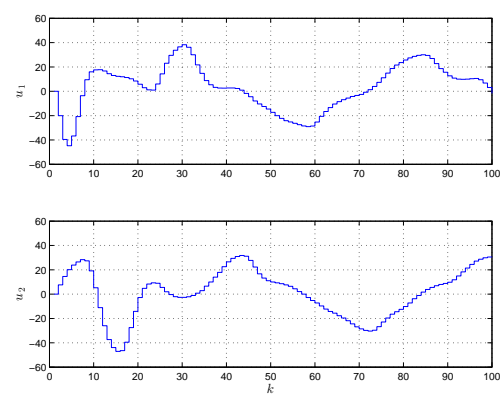

Fig. 2. The generated control input signals using MPC.

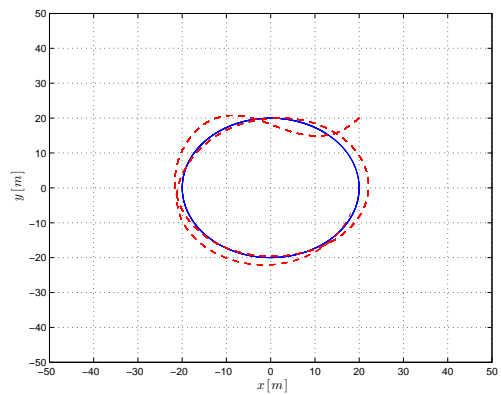

Fig. 3. The obtained tracking performance using regularized MPC. The solid line illustrates the generated reference at each time instant, and the dashed line presents the position of the drone.

(at each time step). The regularization parameter controls the tradeoff between sparsity and the control performance. We, hence, proposed an alternative formulation of the regularized MPC problem, which allowed us to set the trade-off between the control performance and regularization term, in a much more intuitive and efficient manner, by setting the bound over the control performance. This enabled us to produce the sparsest solution possible, by sacrificing a preset amount of control performance.

\section{REFERENCES}

[1] R. Tibsharani, "Regression shrinkage and selection via the lasso," Journal of Royal Statistical Society B (Methodological), vol. 58, no. 1, pp. 267-288, 1996.

[2] H. Ohlsson, L. Ljung, and S. Boyd, "Segmentation of ARX-models using sum-of-norms regularization," Automatica, vol. 46, no. 6, pp. 1107-1111, 2010.

[3] H. Ohlsson, F. Gustafsson, L. Ljung, and S. Boyd, "Smoothed state estimates under abrupt changes using sum-of-norms regularization," Automatica, vol. 48, no. 4, pp. 595-605, 2012.

[4] N. Ozay, M. Sznaier, C. Lagoa, and O. Camps, "A sparsification approach to set membership identification of a class of affine hybrid systems," in 47th IEEE Conference on Decision and Control, Dec. 2008, pp. 123-130.

[5] D. L. Donoho, "Compressed sensing," IEEE Transactions on Information Theory, vol. 52, no. 4, pp. 1289-1306, Apr. 2006.

[6] E. J. Candes, M. B. Wakin, and S. P. Boyd, "Enhancing sparsity by reweighted $\ell_{1}$ minimization," Journal of Fourier Analysis and Applications, special issue on sparsity, vol. 14, no. 5, pp. 877-905, 2008.

[7] K. Åström and B. Bernhardsson, "Systems with lebesgue sampling," in Directions in Mathematical Systems Theory and Optimization, ser. Lecture Notes in Control and Information Sciences, A. Rantzer and C. Byrnes, Eds. Springer Berlin / Heidelberg, 2003, vol. 286, pp. $1-13$.
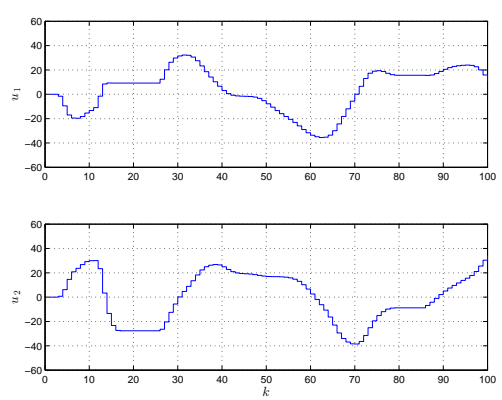

Fig. 4. The generated control input signals using regularized MPC.

[8] H. Ohlsson, F. Gustafsson, L. Ljung, and S. Boyd, "Trajectory generation using sum-of-norms regularization," in Proceedings of 49th IEEE Conference on Decision and Control, Dec. 2010, pp. 540-545.

[9] J. Rawlings, "Tutorial overview of model predictive control," IEEE Control Systems, vol. 20, no. 3, pp. 38-52, Jun. 2000.

[10] J. M. MacIejowski, Predictive Control: With Constraint, ser. Pearson Education. Prentice Hall, 2002.

[11] D. Q. Mayne, J. Rawlings, C. V. Rao, and P. O. M. Scokaert, "Constrained model predictive control: Stability and optimality," $\mathrm{Au}$ tomatica, vol. 36, no. 6, pp. 789-814, 2000.

[12] M. Sznaier, R. Suarez, and J. Cloutier, "Suboptimal control of constrained nonlinear systems via receding horizon constrained control lyapunov functions,", Int. J. Robust Nonlinear Control, vol. 13, no. 3-4, pp. 247-259, 2003

[13] Y. Wang and S. Boyd, "Performance bounds for linear stochastic control," Systems and Control Letters, vol. 58, no. 3, pp. 178-182, 2009.

[14] S. Boyd and L. Vandenberghe, Convex optimization. Cambridge University Press, 2004.

[15] Y. Wang and S. Boyd, "Fast model predictive control using online optimization," IEEE Transactions on Control Systems Technology, vol. 18, no. 2, pp. 267-278, Mar. 2010.

[16] E. J. Candès, Y. C. Eldar, and D. Needell, "Compressed sensing with coherent and redundant dictionaries," CoRR, vol. abs/1005.2613, 2010.

[17] H. Ohlsson, L. Ljung, and S. Boyd, "Segmentation of ARX-models using sum-of-norms regularization," Automatica, vol. 46, no. 6, pp. 1107-1111, 2010.

[18] H. Ohlsson, T. Chen, S. Khoshfetrat Pakazad, L. Ljung, and S. Sastry, "Distributed change detection," 16th IFAC Symposium on System Identification, Brussels, pp. 77-82, 2012.

[19] N. Ozay, M. Sznaier, C. Lagoa, and O. Camps, "A sparsification approach to set membership identification of a class of affine hybrid systems," in Decision and Control, 2008. CDC 2008. 47th IEEE Conference on, Dec, pp. 123-130.

[20] M. Gallieri and J. Maciejowski, "LASSO MPC: Smart regulation of over-actuated systems," in Proc. of the American Control Conference, Montral, Canada, June 2012, pp. 1217-1222.

[21] M. Annergren, A. Hansson, and B. Wahlberg, "An admm algorithm for solving $\ell_{1}$ regularized MPC," in Proceedings of 51st IEEE Conference on Decision and Control, Dec. 2012, pp. 4486-4491.

[22] H. Ohlsson and L. Ljung, "Identification of switched linear regression models using sum-of-norms regularization," Automatica, 2013, to appear.

[23] M. Ehrgott, Multicriteria Optimization. Springer, 2005.

[24] Y. Haimes, L. Lasdon, and D. Wismer, "On a bicriterion formulation of the problems of integrated system identification and system optimization," IEEE Transactions on Systems, Man and Cybernetics, vol. SMC-1, no. 3, pp. 296-297, Jul 1971.

[25] M. Ehrgott and D. Ryan, "Constructing robust crew schedules with bicriteria optimization," Journal of Multi-Criteria Decision Analysis, vol. 11, no. 3, pp. 139-150, 2002.

[26] H. Benson, "Existence of efficient solutions for vector maximization problems," Journal of Optimization Theory and Applications, vol. 26, no. 4, pp. 569-580, 1978.

[27] V. Chankong and Y. Haimes, Multiobjective Decision Making: Theory and Methodology. New York: Elsevier Science Publishing Co., 1983. 